\title{
Riemannian foliations and the kernel of the basic Dirac operator
}

\author{
Vladimir Slesar
}

\begin{abstract}
In this paper, in the special setting of a Riemannian foliation endowed with a bundle-like metric, we obtain conditions that force the vanishing of the kernel of the basic Dirac operator associated to the metric; this way we extend the traditional setting of Riemannian foliations with basic-harmonic mean curvature, where Bochner technique and vanishing results are known to work. Beside classical conditions concerning the positivity of some curvature terms we obtain new relations between the mean curvature form and the kernel of the basic Dirac operator.
\end{abstract}

\section{Introduction}

In the framework of a closed differential manifold endowed with a foliated structure and a bundle-like metric tensor field (i.e. the manifold can be locally described as a Riemannian submersion [18]), the natural differential operators canonically associated to the Riemannian structure can be defined. As in the classical case, they are known to play a crucial role in the study of the geometry of the underlying foliated manifold.

For the so called basic Laplacian which acts on the de Rham complex of basic differential forms (or for the transversal Laplacian, if one consider general differential forms instead of basic forms), the relevant features has been carried out in the last period of time $[2,4,12,17,19,21]$.

Key Words: Riemannian foliations, Dirac bundles, vanishing results.

2010 Mathematics Subject Classification: 57R30, 55R10, 58J50.

Received: August, 2011.

Accepted: February, 2012. 
On the other side, the transversal Dirac operator for Riemannian foliations were introduced in [6]. In the particular case of a Riemannian foliation with basic mean curvature form, this operator is used to defined the basic Dirac operator, which is symmetric, essentially self-adjoint and transversally elliptic [6]. A Weitzenböck-Lichnerowicz formula is also obtained, and in the case when the mean curvature form of the Riemannian foliation is not only basic, but also harmonic, Bochner techniques can be implemented and vanishing results can be obtained [3]. We emphasize the fact that in this specific framework conditions for the vanishing of the basic Dirac kernel are related to curvature type operator, as in the classical case [7], but also to the mean curvature, exhibiting the specific nature of a Riemannian foliated manifold.

Now, concerning the mean curvature form and the way it varies when the bundle-like metric is changed, we refer to $[1,5,16]$. In [5], using a Hodge-type decomposition theorem from [1], the author show that any bundle-like metric can be transformed such that the new bundle-like metric have basic mean curvature form; in fact the transformation leaves invariant the transversal metric and the basic component of the mean curvature form of the initial metric. Furthermore, as an application of stochastic flows in theory of Riemannian foliations, in [16] the author constructs a dilation of the metric which turn it into a metric with basic-harmonic mean curvature.

In [9], the authors recently proved the invariance of the spectrum of the basic Dirac operator with respect to a special class of transformations of the bundle-like metric; more exactly, the metric on $M$ can be changed in any way that leaves the transverse metric on the normal bundle intact. They also used a generalized definition of the basic Dirac operator, defined this time on a general Riemannian foliation. Using this result we can derive a method for studying the spectrum of such Dirac-type operator, as pointed out in [9]; that is, we may assume the bundle like metric is chosen so that the mean curvature is basic-harmonic, the result being therefore pulled back in the general case using [5] and [16]. As an application, the authors finally obtained the eigenvalues estimate for arbitrary Riemannian foliation with bundle-like metric.

In this paper, using the spectral rigidity result [9], we obtain the corresponding version of the classical vanishing result of the kernel on Dirac bundle [7]. Furthermore, we use a Weitzenböck-Lichnerowicz formula for the basic Dirac operator which is different from $[3,6]$. Let us point out that this formula allows one to perform classical Bochner technique directly in the case of a Riemannian foliation with basic, non-necessarily harmonic mean curvature [20]. In the same framework of arbitrary Riemannian foliations, we get a second vanishing condition related this time to the mean curvature vector field.

The second and the third section contained the definitions of the geo- 
metric objects we are dealing with and a presentation of the WeitzenböckLichnerowicz formulas that we employ, while the main results are presented in the last section.

\section{The basic Dirac operator}

Let us consider in what follows a smooth, closed Riemannian manifold $(M, g, \mathcal{F})$ endowed with a foliation $\mathcal{F}$ such that the metric $g$ is bundle-like [18]; the dimension of $M$ will be denoted by $n$. We also denote by $T \mathcal{F}$ the leafwise distribution tangent to leaves, while $Q=T \mathcal{F}^{\perp} \simeq T M / T \mathcal{F}$ will be the transversal distribution. Let us assume $\operatorname{dim} T \mathcal{F}=p, \operatorname{dim} Q=q$, so $p+q=n$.

As a consequence, the tangent and the cotangent vector bundles associated with $M$ split as follows

$$
\begin{aligned}
T M & =Q \oplus T \mathcal{F}, \\
T M^{*} & =Q^{*} \oplus T \mathcal{F}^{*} .
\end{aligned}
$$

The canonical projection operators will be denoted by $\pi_{Q}$ and $\pi_{T \mathcal{F}}$, respectively.

Throughout this paper we will use local vector fields $\left\{f_{a}, e_{i}\right\}$ defined on a neighborhood of an arbitrary point $x \in M$, so that they determine an orthonormal basis at any point where they are defined, $\left\{f_{a}\right\}$ spanning the distribution $Q$ and $\left\{e_{i}\right\}$ spanning the distribution $T \mathcal{F}$.

For the study of the basic geometry of our Riemannian foliated manifold a convenient metric and torsion-free linear connection is the so called Bott connection (see e.g. [21]). If we denote by $\nabla^{g}$ the canonical Levi-Civita connection, then on the transversal distribution $Q$ we can define the connection $\nabla$ by the following relations

$$
\left\{\begin{array}{l}
\nabla_{U} X:=\pi_{Q}([U, X]), \\
\nabla_{Y} X:=\pi_{Q}\left(\nabla_{Y}^{g} X\right),
\end{array}\right.
$$

for any smooth sections $U \in \Gamma(T \mathcal{F}), X, Y \in \Gamma(Q)$. In particular we can associate to $\nabla$ the transversal scalar curvature $S c{ }^{\nabla}$.

We restrict the classical de Rham complex of differential forms $\Omega(M)$ to the complex of basic differential forms, defined as

$$
\Omega_{b}(M):=\left\{\omega \in \Omega(M) \mid \iota_{U} \omega=\mathcal{L}_{U} \omega=0\right\},
$$

where $U$ is again an arbitrary leafwise vector field, $\mathcal{L}$ being the Lie derivative along $U$, while $\iota$ stands for interior product. Considering now the de Rham exterior derivative $d$, it is possible to define the basic operator $d_{b}:=d_{\mid \Omega_{b}(M)}$ 
(see e.g. [1]). Let us notice that basic de Rham complex is defined independent of the metric structure $g$.

One differential form which is not necessarily basic is the mean curvature form. In order to define it, we first of all set $k^{\sharp}:=\pi_{Q}\left(\sum_{i} \nabla^{g}{ }_{e_{i}} e_{i}\right)$ to be the mean curvature vector field associated with the distribution $T \mathcal{F}$, while $k$ will be the mean curvature form which is subject to the condition $k(U)=g\left(k^{\sharp}, U\right)$, for any vector field $U, \sharp$ being the musical isomorphism.

Remark 2.1. It is easy to see that $k\left(k^{\sharp}\right)=\left\|k^{\sharp}\right\|^{2}$.

By Theorem 2.1 in [1], we have the orthogonal decomposition

$$
\Omega(M)=\Omega_{b}(M) \bigoplus \Omega_{b}(M)^{\perp},
$$

with respect to the $C^{\infty}$-Frechet topology. So, on any Riemannian foliation the mean curvature form can be decomposed as the sum

$$
k=k_{b}+k_{o},
$$

where $k_{b} \in \Omega_{b}(M)$ is the basic component of the mean curvature, $k_{o}$ being the orthogonal complement. From now on we denote $\tau:=k_{b}^{\sharp}$.

Using the above notations, at any point $x$ on $M$ we consider the Clifford algebra $C l\left(Q_{x}\right)$ which, with respect to the orthonormal basis $\left\{f_{a}\right\}$ is generated by 1 and the vectors $\left\{f_{a}\right\}$ over the complex field, being subject to the relations $f_{a} \cdot f_{b}+f_{b} \cdot f_{a}=-2 \delta_{a b}, 1 \leq a, b \leq q$, where dot stands for Clifford multiplication. The resulting bundle $C l(Q)$ of Clifford algebras will be called the Clifford bundle over $M$, associated with $Q$. Let us also consider a vector bundle $E$ over $M$ and suppose we have a smooth bundle action

$$
\Gamma(C l(Q)) \otimes \Gamma(E) \longrightarrow \Gamma(E),
$$

denoted also with Clifford multiplication such that

$$
(u \cdot v) \cdot s=u \cdot(v \cdot s),
$$

for $u, v \in \Gamma(C l(Q)), s \in \Gamma(E)$.

As a result, $E$ becomes a bundle of Clifford modules (see e.g. [15]).

If a Clifford bundle $E$ is endowed with a connection $\nabla^{E}$, then $\nabla^{E}$ is said to be compatible with the Clifford action and the Levi-Civitá connection $\nabla$ if

$$
\nabla_{U}^{E}(u \cdot s)=\left(\nabla_{U} u\right) \cdot s+u \nabla_{U}^{E} s,
$$

for any $U \in \Gamma(T M), u \in \Gamma(C l(Q)), s \in \Gamma(E)$, extending canonically the connection $\nabla$ to $\Gamma(C l(Q))$. 
In what follows, we assume the existence of a hermitian structure $(\cdot \mid \cdot)$ on $E$ such that $\left(X \cdot s_{1} \mid s_{2}\right)=-\left(s_{1} \mid X \cdot s_{2}\right)$, for any $X \in \Gamma(Q), s_{1}, s_{2} \in$ $\Gamma(E)$, and a metric connection $\nabla^{E}$, compatible with $C l\left(L_{1}\right)$ action and the connection $\nabla$.

On the above transverse Dirac bundle over $M$, in accordance with [6], we introduce now the transversal Dirac operator,

$$
D_{t r}:=\sum_{a} f_{a} \cdot \nabla_{f_{a}}^{E}
$$

and its restriction to the basic (or holonomy invariant) sections

$$
\Gamma_{b}(E):=\left\{s \in \Gamma_{b}(E) \mid \nabla_{U}^{E} s=0, \text { for any } U \in \Gamma(T \mathcal{F})\right\},
$$

the basic Dirac operator, which is defined using the basic component of the mean curvature form $[6,9]$

$$
D_{b}:=\sum_{a} f_{a} \cdot \nabla_{f_{a}}^{E}-\frac{1}{2} \tau .
$$

Remark 2.2. As we use the connection $\nabla$, considering the definition of $\Omega_{b}(M)$ and $\Gamma_{b}(E)$, the first example is provided by the usual complex of (C- valued) basic forms of $(M, g, \mathcal{F})$.

Remark 2.3. The basic Dirac operator is elliptic in the directions of the distribution $Q$ and essentially self-adjoint with respect to the inner product canonically associated with the closed Riemannian manifold [6].

\section{Weitzenböck-Lichnerowicz type formulas for Rieman- nian foliations}

In $[3,6]$, in the setting of a Riemannian foliation with basic mean curvature form, the authors work out the following Weitzenböck-Lichnerowicz formula, which is a useful tool for studying the spectral properties of the basic Dirac operator (see $[8,11])$

$$
D_{b}^{2}=\sum_{a} \nabla_{f_{a}}^{E *} \nabla_{f_{a}}^{E}-\frac{1}{2} \delta_{b} k_{b}+\frac{1}{4}\|\tau\|^{2}+\mathcal{R} s
$$

where $\mathcal{R}:=\sum_{a<b} f_{a} \cdot f_{b} \cdot R_{f_{a}, f_{b}}^{E}, R^{E}$ being the curvature operator associated with $\nabla^{E}$. The basic de Rham coderivative can be written as [1]

$$
\delta_{b}:=\sum_{a}-\iota_{f_{a}} \nabla_{f_{a}}+\iota_{\tau} .
$$


If we integrate over the manifold $M$, from the relation (3.1) we obtain

$$
\left\|D_{b} s\right\|^{2}=\sum_{a}\left\|\nabla_{f_{a}}^{E} s\right\|^{2}+\int_{M}\left(-\frac{1}{2} \delta_{b} k_{b}+\frac{1}{4}\|\tau\|^{2}\right)(s \mid s)+\int_{M}(\mathcal{R} s \mid s)
$$

Let us define the transversal divergence of a vector field $V \in \Gamma(Q)$,

$$
\operatorname{div}^{\nabla} V:=\sum_{a}\left\langle\nabla_{f_{a}} V, f_{a}\right\rangle
$$

where $\left\{f_{a}\right\}$ is again a transversal orthonormal basis. If the mean curvature is not harmonic, then the term related to de Rham coderivative from (3.2) does not vanish; it can be evaluated as

$$
\begin{aligned}
\delta_{b} k_{b} & =-\sum_{a} \iota_{f_{a}} \nabla_{f_{a}} k_{b}+\iota_{\tau}\left(k_{b}\right) \\
& =-\sum_{a}\left\langle\nabla_{f_{a}} \tau, f_{a}\right\rangle+\|\tau\|^{2} \\
& =-\operatorname{div}^{\nabla} \tau+\|\tau\|^{2}
\end{aligned}
$$

Another useful relation is

$$
\begin{aligned}
\operatorname{div} \tau & =\sum_{a}\left\langle\nabla_{f_{a}} \tau, f_{a}\right\rangle+\sum_{i}\left\langle\nabla_{e_{i}}^{g} \tau, e_{i}\right\rangle \\
& =\operatorname{div}^{\nabla} \tau-\|\tau\|^{2},
\end{aligned}
$$

where $\left\{e_{i}\right\}$ is a local orthonormal frame for the leafwise distribution $T \mathcal{F}$, the basic vector field $\tau$ remaining perpendicular to $T \mathcal{F}$ at any point.

Plugging (3.3) in (3.1), we obtain

$$
\left\|D_{b} s\right\|^{2}=\sum_{a}\left\|\nabla_{f_{a}}^{E} s\right\|^{2}+\int_{M}\left(\frac{1}{2} \operatorname{div}^{\nabla} \tau-\frac{1}{4}\|\tau\|^{2}\right)(s \mid s)+\int_{M}(\mathcal{R} s \mid s)
$$

In the following we hold the assumption of a basic mean curvature differential 1-form $k \equiv k_{b}$.

In [20], the modified connection on the space of basic section $\Gamma_{b}(E)$ is defined in the following manner

$$
\bar{\nabla}_{X}^{E} s:=\nabla_{X}^{E} s-\frac{1}{2}\langle X, \tau\rangle s,
$$

for any $X \in \Gamma(T M)$ and $s \in \Gamma_{b}(E),\langle\cdot, \cdot\rangle$ being our scalar product.

Consequently, the Laplacian of the modified connection can be calculated 


$$
\begin{aligned}
\sum_{a} \bar{\nabla}_{f_{a}}^{E *} \bar{\nabla}_{f_{a}}^{E}= & \sum_{a}\left(-\nabla_{f_{a}}^{E}-\frac{1}{2}\left\langle f_{a}, \tau\right\rangle-\operatorname{div}\left(f_{a}\right)\right)\left(\nabla_{f_{a}}^{E}-\frac{1}{2}\left\langle f_{a}, \tau\right\rangle\right)(3.7) \\
= & -\sum_{a} \nabla_{f_{a}}^{E} \nabla_{f_{a}}^{E}-\sum_{a} \operatorname{div}\left(f_{a}\right) \nabla_{f_{a}}^{E}+\sum_{a} \frac{1}{2}\left\langle\nabla_{f_{a}} f_{a}, \tau\right\rangle \\
& +\sum_{a} \frac{1}{2}\left\langle f_{a}, \nabla_{f_{a}} \tau\right\rangle+\sum_{a} \frac{1}{2}\left\langle f_{a}, \tau\right\rangle \nabla_{f_{a}}^{E}-\sum_{a} \frac{1}{2}\left\langle f_{a}, \tau\right\rangle \nabla_{f_{a}}^{E} \\
& +\frac{1}{4}\|\tau\|^{2}+\sum_{a} \frac{1}{2} \operatorname{div}\left(f_{a}\right)\left\langle f_{a}, \tau\right\rangle \\
= & \sum_{a} \nabla_{f_{a}}^{E *} \nabla_{f_{a}}^{E}+\frac{1}{2} \sum_{a}\left\langle f_{a}, \nabla_{f_{a}} \tau\right\rangle-\frac{1}{4}\|\tau\|^{2} .
\end{aligned}
$$

for any $X \in \Gamma(T M)$ and $s \in \Gamma_{b}(E),\langle\cdot, \cdot\rangle$ being our scalar product.

In the above relation we use the fact that $\nabla_{f_{a}}^{E}-\operatorname{div}\left(f_{a}\right)=\nabla_{f_{a}}^{E *}$ (see e.g. [14]) and the relation

$$
\begin{aligned}
\sum_{a} \operatorname{div}\left(f_{a}\right)\left\langle f_{a}, \tau\right\rangle & =\sum_{a, b}\left\langle\nabla_{f_{b}} f_{a}, f_{b}\right\rangle+\sum_{a, i}\left\langle\nabla_{e_{i}}^{g} f_{a}, e_{i}\right\rangle\left\langle f_{a}, \tau\right\rangle \\
& =-\sum_{a, b}\left\langle f_{a}, \nabla_{f_{b}} f_{b}\right\rangle\left\langle f_{a}, \tau\right\rangle-\sum_{a, i}\left\langle f_{a}, \nabla_{e_{i}}^{g} e_{i}\right\rangle\left\langle f_{a}, \tau\right\rangle \\
& =-\sum_{a}\left\langle\nabla_{f_{a}} f_{a}, \tau\right\rangle-\|\tau\|^{2} .
\end{aligned}
$$

On the other hand, we can obtain the following formula from (3.1) using the fact that $d_{b} k=0$, or from the more general formula stated in Theorem 4 in [14], using the definition of $\Gamma_{b}(E)$ :

$$
\begin{aligned}
D_{b}^{2}= & \sum_{a} \nabla_{f_{a}}^{E *} \nabla_{f_{a}}^{E}-\frac{1}{2} \sum_{a} f_{a} \cdot \nabla_{f_{a}} \tau \\
& -\frac{1}{4}\|\tau\|^{2}+\mathcal{R}
\end{aligned}
$$

Considering (3.7) and (3.8), we get

$$
\begin{aligned}
D_{b}^{2}= & \sum_{a} \bar{\nabla}_{f_{a}}^{E *} \bar{\nabla}_{f_{a}}^{E}-\frac{1}{2} \sum_{a} f_{a} \cdot \nabla_{f_{a}} \tau \\
& -\frac{1}{2} \sum_{a}\left\langle f_{a}, \nabla_{f_{a}} \tau\right\rangle+\mathcal{R} .
\end{aligned}
$$


From here, integrating over the closed manifold $M$, we obtain:

$$
\begin{aligned}
\left\|D_{b} s\right\|^{2}= & \sum_{a}\left\|\bar{\nabla}_{f_{a}}^{E} s\right\|^{2}-\frac{1}{2} \int_{M} \sum_{a}\left\langle f_{a}, \nabla_{f_{a}} \tau\right\rangle(s \mid s) \\
& -\frac{1}{2} \int_{M} \sum_{a}\left(f_{a} \cdot \nabla_{f_{a}} \tau \cdot s \mid s\right)+\int_{M}(\mathcal{R} s \mid s),
\end{aligned}
$$

for any $s \in \Gamma(E)$ where $\|\cdot\|$ is the $L^{2}$ norm associated with the hermitian structure.

We study now the real and the pure imaginary part of $\sum_{a}\left(f_{a} \cdot \nabla_{f_{a}} \tau \cdot s \mid s\right)$. For this we need the following result due to O. Hijazi which is a direct consequence of the properties of Clifford multiplication and hermitian structure [10].

Lemma 3.1. Using the above notations, $\operatorname{Re}\left(f_{a} \cdot f_{b} \cdot s \mid s\right)=0$ for $1 \leq a, b \leq$ $q, a \neq b$.

Now, for any $X, Y \in \Gamma(Q)$, with respect to the orthonormal basis $\left\{f_{a}\right\}$, using the Einstein notations, we can write locally $X=X^{a} f_{a}, Y=Y^{b} f_{b}$. As a consequence

$$
\begin{aligned}
(X \cdot Y \cdot s \mid s) & =\left(X^{a} f_{a} \cdot Y^{b} f_{b} \cdot s \mid s\right) \\
& =-\langle X, Y\rangle(s \mid s)+\sum_{a \neq b} X^{a} Y^{b}\left(f_{a} \cdot f_{b} \cdot s \mid s\right) .
\end{aligned}
$$

Using the above Lemma, we get that $\operatorname{Re}(X \cdot Y \cdot s \mid s)=-\langle X, Y\rangle(s \mid s)$. As a consequence,

$$
\operatorname{Re}\left(\int_{M} \sum_{a}\left(f_{a} \cdot \nabla_{f_{a}} \tau \cdot s \mid s\right) d \mu\right)=-\int_{M} \sum_{a}\left\langle f_{a}, \nabla_{f_{a}} \tau\right\rangle(s \mid s),
$$

and, finally the formula becomes [20]

$$
\left\|D_{b} s\right\|^{2}=\sum_{a}\left\|\bar{\nabla}_{f_{a}}^{E} s\right\|^{2}+\operatorname{Re}\left(\int_{M}(\mathcal{R} s \mid s)\right) .
$$

\section{The Bochner technique for the basic Dirac operator}

In the case when the mean curvature 1-form is not only basic, but also harmonic $\left(\delta_{b} k_{b}=0\right)$, the classical Bochner technique applied to formula $(3.2)$ 
yields vanishing results for harmonic sections of $D_{b}[3,6]$. Unfortunately, for non-harmonic mean curvature the term related to basic de Rham coderivative is difficult to estimate. In turn, using the invariance of the basic Dirac spectrum from [9] and (3.11), in the following we obtain vanishing conditions for the kernel of basic Dirac operator in the setting of general Riemannian foliations.

The standard assumption in this context is the non-negativity of the curvature operator $\mathcal{R}$, that is $(\mathcal{R} s \mid s)_{x}>c(s \mid s)_{x}$ for any $s$, at any point $x \in M$ (see e.g. [7]).

Remark 4.1. Concerning the eigensections of the basic Dirac operator for a foliation with non-negative curvature term $\mathcal{R}$, a characterization can be obtained as a direct application of the Bochner argument for the formula (3.11); that is, any harmonic eigensection with respect to $D_{b}$ must satisfy the equation [20]

$$
\nabla_{X}^{E} s-\frac{1}{2}\langle X, \tau\rangle s=0
$$

for any vector field $X \in \Gamma(T M)$.

As pointed out in the introductory section, the metric change described in [5] leaves the transverse metric and the basic part $k_{b}$ of the mean curvature intact, so the action of the basic Dirac operator and the modified connection on $\Gamma_{b}(E)$ does not changes. As a consequence, the result is extended to arbitrary Riemannian foliations using [5].

In the following, we present conditions which force the vanishing of the set of harmonic eigensections of $D_{b}$.

From now on, by taut foliation we will denote a Riemannian foliation which admits a bundle-like metric with a vanishing mean curvature (see e.g. [1]).

Theorem 4.2. On a Riemannian foliation $(M, g, \mathcal{F})$, if the basic curvature operator $\mathcal{R}$ is nonnegative and furthermore, the foliation is not taut or $\mathcal{R}$ is strictly positive at one point $x \in M,\left((\mathcal{R} s \mid s)_{x}>c(s \mid s)_{x}\right.$ for any $s$, with $c>0)$, then there are no nontrivial harmonic eigensection.

Proof. The result is a direct consequence of [16] and [9]; namely, we consider a metric change as in [16]; consequently we obtain a new bundle-like metric and a new basic Dirac operator which isa a conjugate of the initial Dirac operator, and consequently they have the same spectrum; moreover, the new basic component of the mean curvature is basic-harmonic. As a result, using the formula (3.2), it turns out that in order to have nontrivial eigensection we need $\tau=0, s_{x}=0$ and $\sum_{a}\left\|\nabla_{f_{a}} s\right\|^{2}=0$, so $s$ is parallel with respect to the transversal directions. Concerning the first condition, our foliation needs to be taut. Concerning the last two conditions, as $s$ is a basic section, it is in 
fact parallel on the manifold $M$ with respect to the connection $\nabla$. The result now follows arguing as in the classical case [15]. Applying the spectral rigidity from [9], the result is pulled back in the general case.

Remark 4.3. We emphasize the fact that the tautness of a foliations is related to the basic cohomology of the foliation; indeed, applying a metric change as in [5], the basic cohomology class of $k_{b}$ remains invariant [1]. As the basic cohomology is a topological invariant [13], we have in fact a topological condition for having nontrivial basic Dirac kernel.

Another important feature of the basic Dirac operator is that even in the case of the basic de Rham complex its square do not equals the basic Laplace operator [9], so relations between the kernel and the groups of the basic cohomology complex cannot be obtained via some Hodge-de Rham theorem.

As an example of the above result, we may consider a more specific setting, namely we assume that the foliation $\mathcal{F}$ is transversally oriented and has a transverse spin structure. This means that there exists a principal $\operatorname{Spin}(q)$ bundle $\tilde{P}$ which is a double sheeted covering of the transversal principal $S O(q)$ bundle of oriented orthonormal frames $P$, such that the restriction to each fiber induces the covering projection $\operatorname{Spin}(q) \rightarrow S O(q)$; such a foliation is called spin foliation [9]. Similar to the classical case [15], if we denote by $\Delta_{q}$ the spin irreducible representation associated with $Q$, then one can construct the foliated spinor bundle $S:=\tilde{P} \times_{\operatorname{Spin}(q)} \Delta_{q}$. The hermitian metric on $S$ is now induced from the transverse metric. Also, the lifting of the Riemannian connection on $P$ can be used to introduce canonically a connection on $S$.

This is in fact the classical setting when the curvature term can be calculated explicitly; as the twisted curvature term vanishes (see e.g. [15]), we obtain $\mathcal{R}=\frac{1}{4} S$ cal $^{\nabla}$. As a consequence, for a spin foliation we may obtain the corresponding version in this particular framework of the well known results of A. Lichnerowicz on spin manifolds.

Remark 4.4. In the above particular setting, the tautness condition can be derived directly from a more general result concerning the limiting case of the lower bound problem for the eigenvalues of the basic Dirac operator; this was stated first of all in the case of basic-harmonic mean curvature in [11], and extended to arbitrary Riemannian foliations in [9].

Now, searching for more convenient vanishing condition, let us notice that in the restricted setting of a Riemannian foliations with $\mathcal{R} \equiv 0$ and basic mean curvature, if $\operatorname{div}^{\nabla} \tau>\frac{1}{2}\|\tau\|^{2}$ at any point $x \in M$, then the vanishing result for the set of harmonic sections can also be obtained using (3.5). In the final part of the paper, we show that in fact the following stronger result holds.

Theorem 4.5. If $(M, g, \mathcal{F})$ is a Riemannian foliation with $\mathcal{R} \equiv 0$, and $\operatorname{div}^{\nabla} \tau>$ 0 over the compact manifold $M$, then again $\operatorname{ker} D_{b}$ is trivial. 
Proof. The result can be obtained using the transverse divergence theorem, the non-tautness of the foliations and the above result. In the following we show that it can be also achieved by direct calculation applying the new Weitzenböck-Lichnerowicz formula (3.11).

First of all, let us assume the mean curvature to be a basic form. We define the connection

$$
\bar{\nabla}_{X}^{c} s:=\bar{\nabla}_{X} s+c\langle X, \tau\rangle s,
$$

for the arbitrary real constant $c$.

If we take the square in (4.1) and integrate over the closed manifold $M$, we obtain

$$
\begin{aligned}
\int_{M} \sum_{a}\left|\bar{\nabla}_{f_{a}}^{c} s\right|^{2}= & \int_{M}|\bar{\nabla} s|^{2}+\int_{M} c^{2}\|\tau\|^{2}|s|^{2} \\
& +\int_{M} 2 c \operatorname{Re}\left\langle\bar{\nabla}_{\tau} s, s\right\rangle .
\end{aligned}
$$

For the third term, let us notice that

$$
\begin{aligned}
2 \operatorname{Re}\left\langle\bar{\nabla}_{\tau} s, s\right\rangle & =2 \operatorname{Re}\left\langle\nabla_{\tau} s-\frac{1}{2}\langle\tau, \tau\rangle s, s\right\rangle \\
& =-\|\tau\|^{2}|s|^{2}+2 \operatorname{Re}\left\langle\nabla_{\tau} s, s\right\rangle .
\end{aligned}
$$

Considering the classical formula

$$
\operatorname{div}\left(|s|^{2} \tau\right)=\tau\left(|s|^{2}\right)+|s|^{2} \operatorname{div} \tau
$$

and the fact that the connection $\nabla$ is metric and the underlying manifold $M$ is closed, we obtain using (3.4)

$$
\begin{aligned}
\int_{M} 2 \operatorname{Re}\left\langle\nabla_{\tau} s, s\right\rangle & =\int_{M} 2 \frac{1}{2} \tau\left(|s|^{2}\right) \\
& =-\int_{M} \operatorname{div} \tau|s|^{2} \\
& =-\int_{M}\left(\operatorname{div}^{\nabla} \tau-\|\tau\|^{2}\right)|s|^{2} \\
& =-\int_{M} \operatorname{div}^{\nabla}(\tau)|s|^{2}+\int_{M}\|\tau\|^{2}|s|^{2}
\end{aligned}
$$


The third term from (4.2) can be computed from (4.3) and (4.4):

$$
\int_{M} 2 \operatorname{Re}\left\langle\bar{\nabla}_{\tau} s, s\right\rangle=-\int_{M} \operatorname{div}^{\nabla}(\tau)|s|^{2} .
$$

From here, the formula (4.2) can be finally written in the following way

$$
\int_{M}\left|\bar{\nabla}^{c} s\right|^{2}=\int_{M}\left(|\bar{\nabla} s|^{2}+c^{2}\|\tau\|^{2}|s|^{2}-c \operatorname{div}^{\nabla}(\tau)|s|^{2}\right) .
$$

We put $s \in \operatorname{Ker} D_{b}$. Consequently, using (3.11), the equation becomes

$$
\int_{M}\left|\bar{\nabla}^{c} s\right|^{2}=-c \int_{M}\left(\operatorname{div}^{\nabla}(\tau)-c\|\tau\|^{2}\right)|s|^{2} .
$$

As the manifold $M$ is compact, let us consider $c_{1}<\min _{x \in M} \operatorname{div}_{x}^{\nabla}(\tau)$, and $c_{2}=\max _{x \in M}\|\tau\|^{2}$, with $c_{1}, c_{2}>0$. If we set $c:=\frac{c_{1}}{c_{2}}$, from (4.6) we obtain that $s=0$.

As pointed out before, the metric change described in [5] leaves invariant the transversal metric and the basic component of the mean curvature. On the other side we have

$$
\operatorname{div}^{\nabla} \tau=\sum_{a} \iota_{f_{a}} \nabla_{f_{a}} k_{b}
$$

so both the differential operator and the $k_{b}$ are invariant and the result can be extended to arbitrary Riemannian foliations using [5] and the spectral rigidity result from [9].

\section{References}

[1] J. A. Álvarez López, The basic component of the mean curvature of Riemannian foliations, Ann. Global Anal. Geom. 10 (1992), 179-194.

[2] J. A. Álvarez López, Y.A. Kordyukov, Adiabatic limits and spectral sequences for Riemannian foliations, Geom. and Funct. Anal. 10 (2000), 977-1027.

[3] J. Brüning, F.W. Kamber, Vanishing theorems and index formulas for transversal Dirac operators, AMS Meeting 845, Special Session on Operator Theory and Applications to Geometry, American Mathematical Society Abstracts, Lawrence, KA, 1988. 
[4] M. Craioveanu, M. Puta, Asymptotic properties of eigenvalues of the basic Laplacian associated to certain Riemannian foliations, Bull. Math. Soc. Sci. Math. Roumanie, (NS) 35 (1991), 61-65.

[5] D. Domínguez, A tenseness theorem for Riemannian foliations, C. R. Acad. Sci. Sér. I 320 (1995), 1331-1335.

[6] J. F. Glazebrook, F. W. Kamber, Transversal Dirac families in Riemannian foliations. Commun. Math. Phys. 140(1991), 217-240.

[7] M. Gromov, H. B. Lawson, Spin and scalar curvature in the presence of a fundamental group I, Ann. of Math. 111(1980), 209-230.

[8] G. Habib, Tenseur dimpulsion-énergie et feuilletages, Ph.D thesis (2006), Université Henri Poincaré, Nancy.

[9] G. Habib, K. Richardson, A brief note on the spectrum of the basic Dirac operator, Bull. London Math. Soc. 41 (2009), 683-690.

[10] O. Hijazi, A conformal lower bound for the smallest eigenvalue of the Dirac operator and Killing spinors, Commun. Math. Phys. 104 (1986), 151-162.

[11] S. D. Jung, The first eigenvalue of the transversal Dirac operator. J. Geom. Phys. 39 (2001), 253-264.

[12] F. Kamber, Ph. Tondeur, De Rham-Hodge theory for Riemannian foliations, Math. Ann. 277 (1987), 425-431.

[13] A. El Kacimi Alaoui, M. Nicolau, On the topological invariance of the basic cohomology, Math. Ann. 295 (1993), 627-634.

[14] Y. A. Kordyukov, Vanishing theorem for transverse Dirac operators on Riemannian foliations, Ann. Glob. Anal. Geom. 34 (2008), 195-211.

[15] H. B. Lawson, M. L. Michelsohn, Spin geometry, Princeton University Press, 1989.

[16] A. Mason, An application of stochastic flows to Riemannian foliations, Houston J. Math. 26 (2000), 481-515.

[17] E. Park, K. Richardson, The basic Laplacian of a Riemannian foliation, Amer. J. Math. 118 (1996), 1249-1275.

[18] B. Reinhart, Foliated manifolds with bundle-like metrics. Ann. Math. 69 (1959), 119-132. 
[19] V. Slesar, Weitzenböck formulas for Riemannian foliations, Diff. Geom. App. 27 (2009), 362-367.

[20] V. Slesar, On the Dirac spectrum of Riemannian foliations admitting a basic parallel 1- form, J. Geom. Phys. 62 (2012), 804-813.

[21] Ph. Tondeur, Geometry of Foliations, Birkhäuser, Basel, Boston, 1997.

Vladimir SLESAR,

Department of Mathematics,

University of Craiova,

13 Al.I. Cuza, Craiova, RO-200585, Romania.

Email: vlslesar@central.ucv.ro 